\title{
Wavelet Based Spatial Scaling of Coupled Reaction Diffusion Fields
}

\author{
Sudib K. Mishra ${ }^{1}$, Krishna Muralidharan ${ }^{2}$, Pierre Deymier ${ }^{2}$, \\ George Frantziskonis ${ }^{1,2}$, Srdjan Simunovic ${ }^{3}$, and Sreekanth Pannala ${ }^{3}$ \\ ${ }^{1}$ Civil Engineering \& Engineering Mechanics, \\ University of Arizona \\ ${ }^{2}$ Materials Science \& Engineering, University of Arizona, \\ Tucson, AZ 85721 USA \\ ${ }^{3}$ Computer Science \& Mathematics Division, Oak Ridge National Laboratory, \\ Oak Ridge, TN 37831, USA \\ \{sudib,krishna, deymier, frantzis\}@email.arizona.edu, \\ \{pannalas, simunovics\} eornl.gov
}

\begin{abstract}
Multiscale schemes for transferring information from fine to coarse scales are typically based on some sort of averaging. Such schemes smooth the fine scale features of the underlying fields, thus altering the fine scale correlations. As a superior alternative to averaging, a wavelet based scheme for the exchange of information between a reactive and diffusive field in the context of multiscale reaction-diffusion problems is proposed and analyzed. The scheme is shown to be efficient in passing information along scales, from fine to coarse, i.e. up-scaling as well as from coarse to fine, i.e. down-scaling. In addition, it retains fine scale statistics, mainly due to the capability of wavelets to represent fields hierarchically. Critical to the success of the scheme is the identification of dominant scales containing the majority of useful information. The scheme is applied in detail to the analysis of a diffusive system with chemically reacting boundary. Reactions are simulated using kinetic Monte Carlo (KMC) and diffusion is solved by finite differences. Spatial scale differences are present at the interface of the KMC sites and the diffusion grid. The computational efficiency of the scheme is compared to results obtained by local averaging, and to results from a benchmark model. The spatial scaling scheme ties to wavelet based schemes for temporal scaling, presented elsewhere by the authors.
\end{abstract}

Keywords: Multiscale, wavelets, up-scaling, down-scaling, reaction, diffusion.

\section{Introduction}

Diffusion from a reactive boundary is inherently a spatially and temporally multiscale problem, since the reaction process typically involves spatiotemporal scales quite different than those in the diffusion process. Reaction phenomena involve microscopic spatial and temporal scales, while diffusion involves mesoscopic ones. Thus, coupling of the two processes efficiently is challenging. Recently, the authors and co-workers developed a wavelet based scheme that addresses temporal scaling in 
such problems [1]. The present work complements those schemes efficiently by offering efficient spatial scaling that can be used in conjunction with the temporal one. In fact, it is shown in this paper that a combined spatial and temporal scaling is needed in order to minimize error from the tradeoff between computational efficiency and loss of information due to up and down-scaling in multiscale schemes.

Modern simulation techniques deal with the coupling of the spatial and temporal scales involved in a problem. Multiscale models fall under two general categories, namely sequential, and concurrent. In the former ones, typically, a set of calculations performed at lower scales is used to estimate parameters needed in a model at higher scales. An example is molecular dynamic simulations used to come up with the parameters needed to describe the constitutive behavior of materials and processes at higher scales modeled by finite difference or finite element methods. In the latter ones (concurrent) different models are used in different spatial regions concurrently, while compatibility of the methods at interfaces is enforced or assumed. An example is the simulation of crack propagation, where atomistic simulations are used in the vicinity of the crack tip while continuum finite elements are employed to simulate the far field response.

A general review on multiscale methods is that of [2]. Discipline specific review papers are also available in the literature, e.g. in general chemical engineering [3], surface science [4], reactor technology [5], heat transfer and thermal modeling [6], and works implementing general approaches such as gap-tooth and time stepping [7]. A general multiscale methodology based on wavelets has been examined for both spatial scales [8] as well as temporal scales [9, 10]. These works take full advantage of the inherent capabilities of wavelet analysis to represent objects in a multiscale fashion. The waveletbased approach, termed the compound wavelet matrix method, establishes a communication bridge between phenomena at different scales.

\section{Reaction Diffusion System}

The reaction diffusion system is simulated by utilizing principles of operator splitting, which allows treating the reaction operator separately from the diffusion one. Once the two phenomena are simulated at two different scales the multiscale bridging and passing information from lower to higher scales and getting feedback from higher to lower scales is accomplished through multiscale interfacing. This section presents the details of the reaction and diffusion processes for the problems herein.

The chemical processes are treated under the framework of reaction kinetics. First order reactions are considered in this work, i.e. the reaction rate is proportional to the concentration of the reactant to the first power. For reversible reactions such as

$$
\begin{aligned}
& A \stackrel{k_{A B}}{\longrightarrow} B \\
& B \stackrel{k_{B A}}{\longrightarrow} A
\end{aligned}
$$

the first order rate constants $k_{A B}, k_{B A}$, each of inverse time units, define the reaction kinetics governed by

$$
\frac{d[A]}{d t}=-k_{A B}[A]+k_{B A}[B], \quad \frac{d[B]}{d t}=-k_{B A}[B]+k_{A B}[A]
$$

The stochastic formulation of (2) yielding a KMC process is based on the probability distribution function for reaction events expressed as an exponential 


$$
P\left(R=r_{i}\right)=1-e^{-k[S] \Delta t}
$$

where $P$ is the probability of the event $r_{i}$. Here $k$ denotes the reaction rate constant, $[S]$ denotes concentration, and $\Delta t$ is the reaction time demand (time until the next reaction event will occur). Using the time demand, as is standard in formulation of KMC algorithms, the following equations for forward $(A \rightarrow B)$ and backward reaction $(B \rightarrow A)$ are obtained

$$
\begin{aligned}
t_{A B} & =-\frac{1}{k_{A B}[A]} \ln \left(1-R_{1}\right) \\
t_{B A} & =-\frac{1}{k_{B A}[B]} \ln \left(1-R_{2}\right)
\end{aligned}
$$

where $R_{1}$ and $R_{2}$ are random numbers uniformly distributed between zero and unity. At any time in the simulation the reaction which requires the least time is the one that will occur. Thus, at every KMC iteration step, two random numbers are generated, i.e. $R_{1}, R_{2}$, and $t_{A B}, t_{B A}$ are evaluated based on (4). The minimum of $t_{A B}, t_{B A}$ is the time increment associated with the selected reaction event. Since the chemical processes at the boundary are coupled to the diffusion of species by operator splitting, the uncoupled reaction process was described above. For the same reason, the uncoupled diffusion process is described next, with proper boundary condition at the reactive site.

The governing equations for the diffusion of species on a two dimensional, $x-y$ spatial domain is

$$
\frac{\partial u(x, y, t)}{\partial t}=D\left(\frac{\partial^{2} u(x, y, t)}{\partial x^{2}}+\frac{\partial^{2} u(x, y, t)}{\partial y^{2}}\right)
$$

where $D$ denote the diffusion coefficients, considered constant over the domain, and $u$ denotes concentration of species. A finite difference explicit Euler scheme, first order in time and second order in space, with fixed time steps and fixed spatial discretization is used to solve (5). The stability criteria for the numerical integration process is guaranteed when the Courant condition is satisfied, i.e.

$$
\Delta t \leq\left[\frac{(\Delta s)^{2}}{2 D^{-1}}\right]
$$

where $\Delta t, \Delta s$ denote the time increment and minimum spatial grid size, respectively. In this work, the diffusion of species in the 2-D domain is considered deterministic. The stochastic version of the diffusion process yields a Brownian motion process, not examined herein.

The spatially 2-D model consists of the semi-infinite positive half space (diffusion domain) with chemical reactions taking place at the boundary of the half space (reaction domain). The boundary condition at the reactive site is that both $A$, $B$ are specified by the values evaluated from the reaction kinetics during the operation splitting process. The reflecting boundary condition for the half space is implemented in the finite difference scheme by setting the outgoing flux to zero. At the other end of the discretized diffusion problem (far from the reactive site), the species are absorbed. Simulating an absorbing boundary in a finite difference algorithm is not trivial. However, true absorbing boundary can be simulated using an infinite element in a finite element method. For the present problem this issue is 
tackled, simply, by taking a sufficiently large diffusion domain along diffusion direction $(x)$ so that species do not reach the end within the time frame considered. In a general context, however, appropriate measures such as using infinite finite elements must be implemented. Finally, periodic boundary conditions are assumed for the $(y)$ diffusion direction.

Let $\{R\}_{r}$ denote the spatial "signal" or vector of concentrations along $r$ discrete equidistantly spaced reactive points, $x_{1}, x_{2}, \ldots, x_{r}$. Similarly, let $\{D\}_{d}$ denote the spatial "signal" of concentrations along $d$ discrete equidistantly spaced diffusion nodes, $x_{1}, x_{2}, \ldots, x_{d}$, along the reactive boundary. For a benchmark solution of the reaction diffusion problem $r=d$; in this benchmark solution, no spatial scaling is required, yet the process is computationally cumbersome. For an efficient solution, however, $d \ll r$, thus, the number of diffusion grid nodes along the reactive boundary are less than the number of reaction sites. This is examined within the context of averaging and wavelet based mapping in the following two sections. The averaging, wavelet based, and benchmark schemes are illustrated using the discretization parameters listed in Table 1. The benchmark scheme is the most accurate one, since it uses a diffusion domain discretization at the scale of the reaction sites. For this reason, it is computationally expensive. The wavelet based scheme maps 256 reaction sites to 32 nodes in the diffusion grid, thus there are 8 reactive sites contributing to each node in the diffusion domain. The averaging scheme maps 256 reaction sites to 32 nodes in the diffusion grid.

Table 1. Discretization used in the three schemes

\begin{tabular}{lcccccc}
\hline & \multicolumn{3}{c}{ Diffusion domain } & \multicolumn{2}{c}{ Reaction sites } \\
\cline { 2 - 7 } & Nodes & Nodes & Discretization & Discretization & Reaction & Discretization \\
in $x$ & in $y$ & in $x$ & in $y$ & sites & in $x$ \\
Benchmark & 64 & 256 & 0.125 & 0.1250 & 256 & 0.125 \\
$\begin{array}{l}\text { Wavelet } \\
\text { scheme }\end{array}$ & 64 & 32 & 0.125 & 1.0282 & 256 & 0.125 \\
$\begin{array}{l}\text { Averaging } \\
\text { scheme }\end{array}$ & 64 & 32 & 0.125 & 1.0282 & 256 & 0.125 \\
\hline
\end{tabular}

Isotropic diffusion is considered with a diffusion coefficient $D=0.05$ spaceunits $/ \mathrm{sec}^{2}$. The reaction rate for both forward $(\mathrm{A} \rightarrow \mathrm{B})$ and backward $(\mathrm{B} \rightarrow \mathrm{A})$ reactions is considered the same, 2.5 per sec. Thus the reaction kinetics is solely governed by the reactive species concentration and is biased to the direction of lower species concentration. The initial concentration of species A is 100 and of B is 10 at the reactive boundary, while away from the boundary (diffusion domain) the initial concentrations are zero.

\section{Multiscale Interfacing by Averaging}

Averaging can be used for passing information from fine to coarse scales. Here, the concentrations at the nodes along the reactive boundary belonging to the diffusion 
grid are taken as the mean concentration of the reactive sites contributing to that node, i.e. those in its vicinity. Conversely, these mean concentration fields are taken as the initial concentrations along the reactive boundary for the next KMC step. A simple way to map the reaction "signal" over the diffusion grid is to take the mean field of the contributing reaction sites, yielding two vectors based on operations on $\{R\}_{r}$, i.e.

$$
\left\{\begin{array}{c}
\ldots . . \\
R_{i-2} \\
R_{i-1} \\
R_{i} \\
R_{i+1} \\
R_{i+2} \\
\ldots . .
\end{array}\right\} \rightarrow\left\{\begin{array}{l}
\ldots . . \\
D_{i} \\
\ldots .
\end{array}\right\}, \quad\left\{\begin{array}{c}
\ldots . . \\
\delta_{i-2} \\
\delta_{i-1} \\
\delta_{i} \\
\delta_{i+1} \\
\delta_{i+2} \\
\ldots . .
\end{array}\right\}
$$

where $\{\delta\}_{r}$ denotes the deviators (residuals) from the mean field at the reaction sites, and $\{D\}_{r}$ denotes the mean concentrations at the diffusion nodes. Based on averaging,

$$
\{D\}_{d}^{t}=[H]_{d, r}\{R\}_{r}^{t}
$$

holds, with superscript $t$ denoting evaluation at time t, and matrix $H$ of dimensions $d, r$, is circulant, expressed as

$$
[H]=\left[\begin{array}{cccccccccc}
c_{i} & c_{i+1} & c_{i+2} & 0 & 0 & 0 & 0 & 0 & c_{i-2} & c_{i-1} \\
c_{i-1} & c_{i} & c_{i+1} & c_{i+2} & 0 & 0 & 0 & 0 & 0 & c_{i-2} \\
0 & 0 & 0 & \ldots & \ldots & \ldots & 0 & 0 & 0 & 0 \\
c_{i+2} & 0 & 0 & 0 & 0 & 0 & c_{i-2} & c_{i-1} & c_{i} & c_{i+1} \\
c_{i+1} & c_{i+2} & 0 & 0 & 0 & 0 & 0 & c_{i-2} & c_{i-1} & c_{i}
\end{array}\right]_{d, r}
$$

The coefficients in $H$ can be set to appropriate weights for evaluating the mean field. In a way, $H$ acts as a multiscale operating matrix, and this will become more evident in the sequence where the wavelet based scaling is described. The deviators from the mean field are expressed as

$$
\{\delta\}_{r}=\{R\}_{r}-[M]_{r, d}\{D\}_{d}=\{R\}_{r}-[M]_{r, d}[H]_{d, r}\{R\}_{r}
$$

where matrix $M$ maps the $d$ diffusion grid nodes along the reactive boundary to the $r$ reactive sites, and has a sort of unit matrix structure. Thus a stochastic species concentration spatial signal can be decomposed into a mean field which is sent across to coarser scales, i.e. to the diffusion grid, which diffuses the species, and the residual part which contains information on the correlation structure among the individual sites. However, for a deterministically reactive boundary, all sites are reacting equivalently so the residual vector is null in that case. Statistically, all of them are fully correlated. Since mean field in this case is the entire reaction field and the residual part is zero, such a deterministic system should behave as if there is no spatial scaling. This fact will be used in the sequence for validating the implementations. 
Up-scaling maps the reaction sites to the diffusion grid, where species are allowed to diffuse over a time period that covers several reaction time steps. The reason for this is that the characteristic time scale for reaction is orders of magnitude lower than that of the diffusion time scale. An analytical estimate of the number of intermediate KMC steps to be covered between two successive diffusion steps is provided. At the end of the diffusion time step, the species concentrations as they appear on the diffusion grid need to be mapped back onto the reactive sites. This step, crucial to the success of the simulation is called down-scaling (not to be confused to "up-sampling" which is a terminology used mostly in signal processing), or backward feedback from diffusion grid to the reaction sites. Within the averaging framework, this is done by first assigning the updated mean field of concentrations from the diffusion grid to its contributing reactive sites and then adding to them the residuals $\delta$. This operation is expressed as (constituting an explicit scheme)

$$
\{R\}_{r}^{t+1}=[M]_{r, d}^{t}\{D\}_{d}^{t}+\{\delta\}_{r}^{t}
$$

where superscript $t+1$ indicates the time at one diffusion time step after time $t$.

\section{Multiscale Interfacing Using Wavelets}

Here, analogously to the averaging scheme, the concentration at the reactive sites represented through vector $\{R\}_{r}$ are to be up-scaled to the diffusion grid nodes, thus yielding vector $\{D\}_{d}$. Let $W_{R}(s, x)$ denote the wavelet transform of $\{R\}_{r}$, expressed in terms of scale $s$ and spatial coordinate $x . W_{R}(s, x)$ is decomposed as

$$
W_{R}(s, x)=f_{R}\left(s_{0}, \Delta x\right) \oplus f_{R}\left(s_{1}, \Delta x\right) \oplus f_{R}\left(s_{2}, 2^{1} \Delta x\right) \oplus \ldots \oplus f_{R}\left(s_{n}, 2^{n-1} \Delta x\right)
$$

where $f_{R}\left(s_{i}, 2^{i-1} \Delta x\right), i=1, . ., n$ denote the wavelet transform at scale $s_{i}$ and sampling interval $2^{i-1} \Delta x$, and $f_{R}\left(s_{0}, \Delta x\right)$ denotes the transform at the coarsest scale using the scaling function (thus it appears as if there are two wavelet decompositions at the first level, however one is that of the scaling function and the other of the wavelet in (12). Biorthogonal splines of so-called order $[10,4]$ were used in this work. It is noted that for vector $\{R\}_{r}$ there are $n=\log _{2} r-2^{2}$ number of scales. In (12), $\oplus$ implies scalewise association in the wavelet analysis formality. This hierarchical decomposition can be used to up-scale $\{R\}_{r}$ to the diffusion grid by using only the relevant few dominant scales in wavelet domain and truncating the remaining scales. Since the spatial resolution of the diffusion grid is smaller than that of the reaction sites, it may not be possible to up-scale $\{R\}_{r}$ and also have the same level of resolution as in $\{D\}_{d}$. In the wavelet domain this translates to having coarser resolutions than those dictated by the sampling interval at the corresponding scale. Thus, if there are $d$ number of points in the diffusion grid desired to have $\Delta x_{d}$ resolution then out of $n=\log _{2} r-2^{2}$ available scales at the reaction sites only $m=\log _{2} d-2^{2}$ scales, 
which preferably should all belong to the dominant ones, should be retained. Then, the wavelet transform of $\{D\} \mathrm{d}, W_{R}(s, x)$ can be written as

$$
W_{D}(s, x)=f_{R}\left(s_{0}, \Delta x\right) \oplus f_{R}\left(s_{1}, \Delta x\right) \oplus f_{R}\left(s_{2}, 2^{1} \Delta x\right) \oplus \ldots \oplus f_{R}\left(s_{m}, 2^{m-1} \Delta x\right)
$$

where the notation is the same as in (12). By truncating the coefficient at higher scales, it is natural to query whether the energy norm of $\{R\}_{r}$ is preserved. This partially guaranteed by the dominant scale representation of the field. However, truncation of finer scales introduces loss/gain of energy contained in those higher wavelet sub bands. But for all practical purposes the total energy (or mean) remains same; in addition the basic correlation structure is transferred through these scales of dominance. Relevant results are presented in subsequent section.

With the species concentration up-scaled from the reaction sites to the diffusion grid, it is convenient and efficient to solve the diffusion equations in the wavelet domain, until the next, i.e. down-scaling step, described below. Since the wavelet transform is linear, it is permitted to carry over the diffusion equation on the wavelet domain and then back transform it to the physical domain. With the bridging of scales along the $y$ coordinate, the diffusion equation (5) is transformed, with respect to the spatial axis $y$ only in this case, into the wavelet domain, yielding

$$
\frac{\partial W_{y}[u(x, y, t)]}{\partial t}=D\left[\frac{\partial^{2} W_{y}[u(x, y, t)]}{\partial x^{2}}+\frac{\partial^{2} W_{y}[u(x, y, t)]}{\partial y^{2}}\right]
$$

where $W_{y}$ denotes the wavelet transform in the $y$ direction only. Equation (14) can be written as

$$
\frac{\partial u\left(x, W_{y}, t\right)}{\partial t}=D\left[\frac{\partial^{2} u\left(x, W_{y}, t\right)}{\partial x^{2}}+\frac{\partial^{2} u\left(x, W_{y}, t\right)}{\partial y^{2}}\right]
$$

thus expressing $u$ as a function of $x, t$, and the wavelet transform of spatial coordinate $y$. The inverse wavelet transform yields $u$ in the $x, y, t$ domain, i.e.

$$
u(x, y, t)=W_{y}^{-1}\left[u\left(x, W_{y}, t\right)\right]
$$

Finally, it is mentioned that the scales considered in (14)-(16) are the dominant ones.

Similarly to the steps in the averaging scheme, after the diffusion step, $\{D\}_{d}$ is down-scaled to $\{R\}_{r}$ thus providing feedback to the reaction sites from the diffusion grid. The wavelet transform of $\{D\}_{d}$ expressed in (14) contains $m=\log _{2} d-2^{2}$ scales, while $n=\log _{2} r-2^{2}$ scales are required to define the wavelet transform of $\{R\}_{r}$. Since $n>m$, the information in the "lacking" scales, i.e. the finer ones, is obtained from the previous time step. This saliently assumes that the fine scale fluctuations of the concentrations remain invariant or change only a little between two successive up- and down-scaling operations and small diffusion time steps. Given the dominant scales described above, this is a reasonable assumption that does not introduce measurable error. Then, after down-scaling, the wavelet transform of $\{R\}_{r}, W_{R}(s, x)$ is expressed appropriately. 


\section{Results}

Before proceeding to present any results the first thing is to check for the conservation. Conservation is one necessary condition that must be satisfied for any multiscale schemes. We check the mass conservation through counting the total number of species present in the system in kinetic evolution time steps. The relevant evolution shows that, with varying level of spontaneous fluctuations, mass is conserved in both the multiscale schemes.

The parameters listed in TABLE 1 are used for three different schemes. From these, it can be concluded that all three schemes show obvious fluctuations in the kinetic evolution of species, which is due to the KMC reaction process. However, the fluctuations in both the wavelet and averaging schemes are reduced, as compared to the benchmark model. One reason for this is the intermediate time steps. Another reason for the wavelet scheme is that by only considering the dominant scales in the wavelet transform, small scale fluctuations are smeared out. Such smearing is more pronounced in the averaging scheme, since, even though it is incapable of recognizing dominant information, each averaging yields a spatially flat reaction field.

The efficiency of the multi scale schemes depends on their capability to accurately retain temporal as well as spatial information on the concentrations. Thus, along with the temporal evolution the spatial distribution of concentrations should be studied; for compactness, such spatial maps are not shown here.

It can be concluded that, as expected, both the averaging and wavelet spatial scaling schemes compromise the fine scale fluctuations for the gain in computational efficiency. However, it will be shown in the sequence that the wavelet scheme provides superior information as compared to the averaging scheme. The ensemble statistics of the diffusion profile along the diffusion field show that while the ensemble mean values are captured accurately by the multiscale schemes, the standard deviations are not captured as accurately by the averaging scheme as are by the wavelet one. In particular, the averaging scheme overestimates the standard deviations whereas the wavelet scheme underestimates them. The benchmark model is thus sandwiched between the two. The enhanced standard deviation values result from the sharp peaks present in the averaging model. The exclusion of ultra fine scales in wavelet filtering is responsible for a lower standard deviation of the fluctuations along reactive sites. However by increasing the number of wavelet scales in up- and down-scaling brings increasingly more fluctuations.

Another important measure is the capturing of correlation structures by the multiscale schemes. Even though the KMC reactions are uncorrelated, diffusion of species in the $y$-direction introduces correlations. In order to examine whether the multiscale schemes are capable of capturing such correlations, an enhanced correlation on the concentrations along the reactive sites is imposed. Even though these do not contribute to the physics of the problem, they do help understand the effect of multiscaling in detail.

Figure 1 shows the spatial autocorrelation of concentrations along the reactive sites ( $y$-direction, at $x=0$ ) as it results from the three schemes. The benchmark scheme has the correlation structure as it results from the diffusion processes. The wavelet scheme is capable of capturing the spatial correlations by even including only first two 

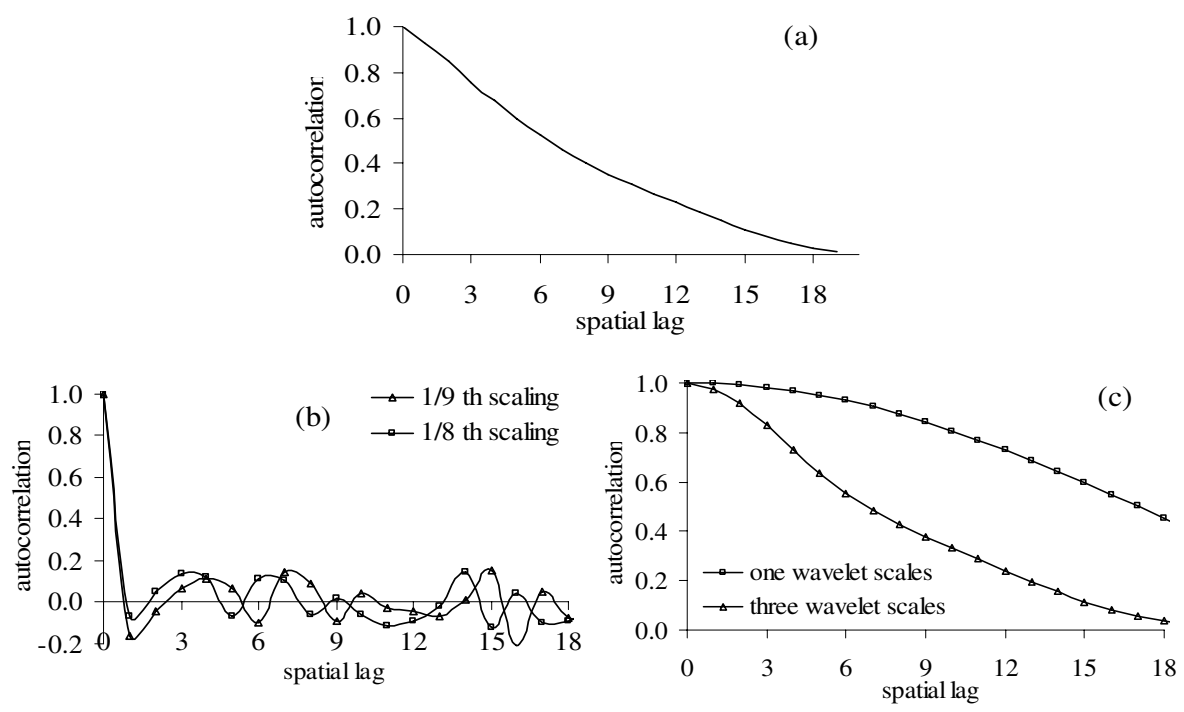

Fig. 1. The autocorrelation function resulting from (a) benchmark, (b) homogenization, (c) wavelet scheme. The scaling in the homogenized and wavelet schemes is defined as $S=\left(\log _{2} r / \log _{2} d\right)$ where $r$ is the number of reaction sites, and $d$ is the number of diffusion sites.

coarsest scales (i.e. the scales containing the scaling coefficients and the next finer scale containing the coarsest wavelet coefficients). With three (or more) scales the autocorrelation structure from the wavelet scheme is practically identical to the one from the benchmark. To the contrary the averaging scheme is incapable of capturing the correlations.

\section{Conclusions}

The spatial scaling wavelet scheme presented herein is offered as a complement to the wavelet based temporal multiscaling presented elsewhere. Even though the spatial multiscaling offers reduced CPU time demand while providing reasonable accuracy, a combined temporal and spatial scaling is required to simulate problems as the present reaction diffusion system efficiently, i.e. representing the behavior accurately at all spatiotemporal scales. An averaging scheme may offer a simple alternative to the wavelet one, yet its efficacy is inferior, especially with respect to correlations.

\section{Acknowledgements}

This research is sponsored by the Mathematical, Information, and Computational Sciences Division; Office of Advanced Scientific Computing Research; U.S. Department of Energy with Dr. Anil Deane as the program manager. The work was partly performed at the Oak Ridge National Laboratory, which is managed by 
UT-Battelle, LLC under Contract No. De-AC05-00OR22725. Discussions with M. Syamlal, T. J. O'Brien, and D. Alfonso of the National Energy Technology Laboratory (NETL), C.S Daw and P. Nukala of Oak Ridge National Laboratory, Rodney Fox and Z. Gao of Iowa State University have been very useful.

\section{References}

1. Frantziskonis, G., Mishra, S.K., Pannala, S., Simunovic, S., Daw, C.S., Nukala, P., Fox, R.O., Deymier, P.A.: Wavelet-based spatiotemporal multi-scaling in diffusion problems with chemically reactive boundary. Int. J. Multiscale Comp. Eng. 4, 755-770 (2006)

2. Vvedensky, D.D.: Multiscale modelling of nanostructures. J. Phys. Cond. Matter 16, R1537-R1576 (2004)

3. Marin, G.B. (ed.):Multiscale Analysis. Advances in Chemical Engineering, book series, vol. 30, pp. 1-309 (2005)

4. Dollet, A.: Multiscale Modeling of CVD Film Growth-a Review of Recent Works. Surface and Coatings Technology, 177-178, 245-251 (2004)

5. Hsiaotao, T., Jinghai, L.I.: Multiscale Analysis and Modeling of Multiphase Chemical Reactors. Advanced Powder Technol. 15, 607-627 (2004)

6. Murthy, J.Y., Narumanchi, S.V.J., Pascual-Gutierrez, J.A., et al.: "Review of Multiscale Simulation in Submicron Heat Transfer. Int. J. Multiscale Computational Engineering 3, 5-31 (2005)

7. Vasenkov, A.V., Fedoseyev, A.I., Kolobov, V.I., et al.: Computational Framework for Modeling of Multi-scale Processes. Computational and Theoretical Nanoscience 3, 453 458 (2006)

8. Frantziskonis, G., Deymier, P.A.: Wavelet Methods for Analyzing and Bridging Simulations at Complementary Scales - the Compound Wavelet Matrix and Application to Microstructure Evolution. Modelling Simul. Mater. Sci. Eng. 8, 649-664 (2000)

9. Frantziskonis, G., Deymier, P.: Wavelet-based Spatial and Temporal Multiscaling: Bridging the Atomistic and Continuum Space and Time Scales. Phys. Rev. B 68, 024105 (2003)

10. Muralidharan, K., Mishra, S., Frantziskonis, G., Deymier, P.A., Nukala, P., Simunovic, S., Pannala, S.: The dynamic compound wavelet matrix for multiphysics/multiscale problems. Phys. Rev. E 77, 026714 (2008) 